\title{
Quality of Life and Psychological Aspects of Diabetes
}

\section{Nafiaa $H^{*}$, Benchikhi L and Ouanass A \\ Arrazi psychiatric hospital, Mohammed V University, Morocco}

*Corresponding author: Nafiaa Hind, Arrazi psychiatric hospital, Mohammed V University, Faculty of Medecine and Pharmacy of Rabat, Sale, Morocco, Tel: +212671859998; E-mail: hind.naf@gmail.com

\section{Conceptual Paper}

Volume 1 Issue 1

Received Date: September 08, 2017

Published Date: October 13, 2017

DOI: $10.23880 / \mathrm{mhrij}-16000108$

\section{Abstract}

Diabetes occupies an important rank among chronic diseases. Its prevalence and severity increase significantly of both somatic and psychic consequences that impairs people's quality of life. According to the International Diabetes Federation (IDF), in 2035, diabetes will affect 592 million people making it one of the leading causes of disability and death world wide. The prevalence of diabetes will increase then from $8.3 \%$ to $10.1 \%$ of the global population. The frequent use of insulin injections, the blood sugar control and treatment of hyperglycemia and hypoglycemia are all elements that are part of a diabetic daily life. This affects badly the psychological health. During recent decades, clinicians and researchers have gradually explored the psychological aspects of diabetes. A study in France of a sample of 50 diabetic patients found that $88 \%$ of participants reported that their life would be better if they were not diabetic. The items of quality of life most affected are: the overall quality of life, diet, psychological aspect, body image and self-esteem. As for health, diabetics often report physical and mental limitation, loss of energy, reduced social activities and entertainment, a frequency of occurrence of neuropathic pain and therefore an impaired general condition. The counseling can improve diabetic quality of life, treatment compliance, limit the patient's disability and help maintain its autonomy. The psychological effects can be kind of depression, anxiety, addictive behavior, personality disorders with regression, relationship difficulties and even suicidalideation. Psycho education is the most important step of diabetic patient counseling: explain the disease to the patient's family, its etiology, development, prognosis, therapeutic principles and possible side effects of treatment. Further more, a device that aims to improve self-esteem, to protect against the effects caused by the illness, and the perception that the subject has need to be developed. These negative effects are expressed in physical forms, physiological, or behavioral, evolving in terms of the degree of acceptance and disease control. This program will prevent and limit the effects of stress experienced daily, and there by improve the quality of life of diabetic patients.

Keywords: Diabetes; Quality of Life; Psychology; Management; Stress; Depression 


\section{Mental Health \& Human Resilience International Journal}

\section{Introduction}

Diabetes is a serious life-long health condition that occurs when the amount of glucose (sugar) in the blood is too high because the body can't use it properly. If left untreated, high blood glucose levels can cause serious health complications.

A blood glucose test is performed in a laboratory for medical analysis. Diabetes is known to occur when fasting blood glucose is equal to or greater than $1.26 \mathrm{~g} / \mathrm{l}$ twice or $2 \mathrm{~g} / \mathrm{l}$ or more at any time of day.

\section{Diabetes and Heredity}

The impact of heredity differs depending on the type of diabetes. If both parents are affected, the risk of having diabetes increases to $70 \%$. It is only $5 \%$ in type 1 diabetes, more precisely $6 \%$ if the father is diabetic, versus $2-3 \%$ if it is the mother (but 30\% if both parents are).

It is therefore useful to build a genealogical tree to identify the members of the diabetic family and to know its genetic heritage.

\section{Types of Diabetes}

There are two main types of diabetes: Type 1 and Type 2. They're different conditions, caused by different things, but they are both serious and need to be treated and managed properly.

\section{Type 1 Diabetes}

Type 1 diabetes, once called insulin-dependent diabetes (IDDM), is usually found in young people: children, adolescents or young adults.

\section{Symptoms of Type 1 Diabetes}

Symptoms are usually: intense thirst, abundant urine, rapid weight loss. It results from the disappearance of the beta cells of the pancreas causing a total deficiency in insulin. The body no longer recognizes these beta cells and destroys them. It is said that type 1 diabetes is an autoimmune disease. Glucose that cannot enter cells returns to the bloodstream. The level of glucose in the blood then rises.

\section{Causes of Type 1 Diabetes}

It is not known why this destruction of the islets of Langerhans occurs, why in some people and not in others. There is a genetic predisposition (familial) but the other causes are poorly known. The environment would also play a role.

\section{Management of type 1 diabetes}

Since the body no longer manufactures insulin at all, the only current treatment is the intake of insulin: Either in the form of injections (injection of insulin with a syringe or a pen), or with an insulin pump (pump treatment), a portable or implantable device for administering insulin continuously.

\section{Type 2 Diabetes}

Type 2 diabetes usually occurs in people over 40 years of age. Overweight, obesity and lack of physical activity are risk factors of type 2 diabetes in genetically predisposed individuals. Slow and painless, the development of type 2 can go unnoticed for a long time: it is estimated that an average of 5 to 10 years relapses between the onset of the first hyperglycemia and the diagnosis.

\section{Etiopathogenesis of Type 2 Diabetes}

In type 2 diabetes, previously called non-insulin dependent (DNID), the process is different from that of type 1 diabetes. Two anomalies are responsible for hyperglycemia:

The pancreas always produces insulin but not enough, in relation to blood sugar: it is insulinopenia; Either this insulin acts badly, it is called insulin resistance, Insulin can no longer regulate blood glucose and this resistance gradually depletes the pancreas which eventually no longer ensures sufficient insulin production. These two mechanisms mean that glucose does not penetrate the cells of the body and remains in the bloodstream. The level of glucose in the blood is not regulated by insulin.

\section{Causes of Type 2 Diabetes}

There is not one specific cause but a set of supporting factors:

A genetic origin: the family factor is quite preponderant. A similar history of diabetes is common in the family; An unbalanced diet, lack of physical activity, overweight

\section{Management of Type 2 Diabetes}

The treatment of diabetes aims at obtaining the best possible glycemic control, thus to the glycated hemoglobin (HbA1c) closest to the normal values, in order to avoid disabling complications in the long term, but also the severe hypoglycemic short term. 


\section{Mental Health \& Human Resilience International Journal}

It is initially treated with hygiene-detox measures, followed by the use of oral and / or injectable antidiabetic treatments which are only optimally effective if they are combined with a balanced diet and sports. Since type 2 diabetes is a progressive disease, after the progressive increase of antidiabetics (therapeutic escalation), insulin injections will be proposed in addition to the patient when insulin deficiency will be too large.

\section{Diabetes and Depression: Is There Any Link?}

Depression and diabetes are two diseases that need to be considered at the same time .But first, we need to define Depression through DSM criteria:

DSM 5 Criteria for Major Depressive Disorder (MDD) [1]:

- Depressed mood or a loss of interest or pleasure in daily activities for more than two weeks.

- Mood represents a change from the person's baseline.

- Impaired function: social, occupational, educational.

- Specific symptoms, at least 5 of these 9, present nearly every day:

1. Depressed mood or irritable most of the day, nearly every day, as indicated by either subjective report

(e.g., feels sad or empty) or observation made by others (e.g., appears tearful).

2. Decreased interest or pleasure in most activities, most of each day

3. Significant weight change $(5 \%)$ or change in appetite

4. Change in sleep: Insomnia or hypersomnia

5. Change in activity: Psychomotor agitation or retardation

\section{Fatigue or loss of energy}

7. Guilt/worthlessness: Feelings of worthlessness or excessive or inappropriate guilt

8. Concentration: diminished ability to think or concentrate, or more indecisiveness

9. Suicidality: Thoughts of death or suicide, or suicide plan

The causes of the link between diabetes and depression are not yet clear. However, stress could be implicated, as cortisol, one of the stress hormones, also contributes to the way our body consumes sugar and how it stores fat, which is important in the development of diabetes. Besides, other, less innovative relationships are mentioned by other specialists. Indeed, a person who has diabetes has good reason to be depressed since this disease, although it can now be managed successfully, remains a chronic condition that often has an impact on the quality of life.
On the other hand, people who suffer from depression tend to take less care of their health, which increases the risk of suffering from diabetes. It should also be said that with two diseases as frequent as diabetes and depression, a certain amount of people who suffer from both should be expected. As in [2], a cross-sectional study, conducted in Hertfordshire County, England, assessed the relationship between depression and anxiety score (Hospital Anxiety and Depression score, HAD-D), diabetes and other metabolic variables (including fasting blood glucose and insulin levels and 30 and $120 \mathrm{~min}$ after oral loading of $75 \mathrm{~g}$ glucose during an oral hyperglycemia test [PGHO]), in 1,577 men and 1,418 women, aged 59 to 73 years (mean age: 66 years). Diabetes was known in 182 participants and diabetes was diagnosed in 249 people in the study, a total of $14.6 \%$ of people with diabetes. The HAD-D score ranked 37 participants as having "probable" depression and 124 as having "possible" depression. The diagnosis of "likely" depression was associated with a 3.9fold higher risk of diabetes in men and 51\% higher in women, and a higher prevalence of diabetes in both sexes. The fact that patients were treated or not by antidepressant did not change the risk.

Therefore, the authors conclude that this study supports the hypothesis that depression is likely to increase the risk of diabetes, and that general practitioners are justified in seeking diabetes in people with depression and vice versa. A prospective study [3] of a cohort of 4,184 people with Type 2 diabetes, followed by general practitioners in Washington State, USA, assessed depression (by the Patient Health Questionnaire, HQ-9) when they were included in the study between 2000 and 2002, and Classified them according to the result in "safe from depression", "minor depression" or "major depression". As is usually the case, people with diabetes suffering from major depression were more likely to be women, were slightly younger and treated with insulin, had higher HbA1c and more medical comorbidities than diabetics Free from depression or with minor depression. It was investigated whether there was a correlation with the causes of death, by studying the death register of this state until 2007. During this period, $428(12.9 \%)$ of depression-free subjects died, 88 (17.8\%) of those with major depression and 88 (17.8\%) of those suffering from a form of minor depression.

Causes of death were grouped into cardiovascular disease $(42.7 \%$ of deaths), cancer $(26.9 \%)$ and other causes $(30.5 \%)$, the most frequent being infections, dementia, End-stage renal disease and chronic obstructive pulmonary disease. After adjusting for the different variables that could influence the risk of death (demographic and clinical characteristics, lifestyle), the 


\section{Mental Health \& Human Resilience International Journal}

presence of major depression at baseline was significantly associated with a higher risk of death from any cause (increased risk of 52\%) and mortality from causes other than cardiovascular or cancer (risk x 2.15). Among those with a minor form of depression at baseline, the patterns were identical but not significant.

The conclusion of the study is that beyond the wellknown increased risk of cardiovascular death, patients with diabetes, when also suffering from major depression, have a risk of death from any cause substantially increased, perhaps reflecting a worse lifestyle, poor adherence to diabetes, or inadequate medical management of other associated illnesses or depression itself. As in [4], a prospective study, covered a cohort of 4,623 people with Type 2 diabetes, followed by general practitioners United States, and began in 2005-2007. They were followed during 5 years.

Associated diseases, severe microvascular complications (blindness, chronic renal failure, kidney dialysis and amputation) and macrovascular (myocardial infarction, stroke, cardiovascular revascularization) related to diabetes and the causes of death were analyzed. After adjustment for various criteria (demographic, clinical, severity and diabetes management), the presence of major depression was associated with a significantly increased risk of severe, microvascular (36\%) and macrovascular (24\%) Compared to DT2 patients without depression.

This study, like many others, highlights the negative association between depression and diabetes, patients with depression have an increased risk of developing diabetes and its complications, and those with diabetes, severe life-threatening complications, such as vision impairment or renal failure requiring dialysis, are more likely to be affected by depression, or a worsening of it if it exists beforehand. In another prospective study [5], where the authors evaluated whether a clinically significant depressive state increased the risk of diabetes Zaragoza Dementia and Depression Project, was carried out in a large representative population of 4,803 people aged 55 and over (average age 72-73 years), randomly selected (20.5\% refusal) among those living in Saragossa (Spain) at the time of the last census, that is to say 3,521 subjects included after exclusion of the study of persons already suffering from diabetes or having any kind of dementia. It took place in three successive waves: inclusion, then detailed interrogations 2.2 years and 4.7 years later on average.

Different scales and scores were used to characterize depressive states, as well as to assess diabetes risk factors. At baseline, 379 subjects were classified as depressed and 3,142 as depressed. During the mean follow-up of 4.7 years, 163 new cases of diabetes were diagnosed, $25(6.6 \%$ of subjects $)$ in those initially classified as depressed, and 138 (4.4\% of subjects) Suffering from depression; The annual incidence of new cases of diabetes was higher in subjects initially classified as depressed than in those without depression (19.7 cases / 1,000 people per year vs. 12.4 cases / 1,000 people per year, respectively).

The risk of developing diabetes in people with depression appeared to be significantly increased (by $65 \%$ ) in depression compared to those without depression even after adjusting for different risk factors for diabetes. The rate of diabetes due to depression was estimated to be $6.9 \%$. An increased risk of diabetes is associated with certain characteristics of depression: nonsevere depression, persistent depression, untreated depression. On the other hand, antidepressant therapy was not associated with an increased risk of diabetes.

The authors emphasize that these depressive characteristics, associated with the increased risk of diabetes, are frequently present in the elderly; it may be useful to detect and / or try to prevent them, although it remains to be demonstrated whether this would result in a reduction in the risk of diabetes in this population.

\section{Diabetes and Stress}

In case of severe stress, the body secretes stress hormones like adrenaline. They cause a narrowing of the blood vessels and an increase in the blood pressure: we are then more vigilant and more effective. At the same time, these hormones release the sugar from the cells and the blood sugar increases so that the body can have more energy at its disposal.

Under the influence of stress, the brain and muscles burn more sugar than normal. If it is moderate, this process is completely harmless to health. But if the balance in the body is disrupted by stress, this can trigger the onset of diabetes. Of course, external circumstances do not always influence stress. However, regular walks, adequate sleep and the following relaxation exercises can help lower adrenaline levels and help you regain serenity.

The onset of type 2 diabetes is very much related to lifestyle. A Swedish study highlights the role of stress. Researchers at the University of Göteborg (Sweden) have followed 6,828 men since 1970 , while the subjects were between 47 and 56 years of age. These patients completed questionnaires to assess their stress, while they had no 


\section{Mental Health \& Human Resilience International Journal}

previous history of diabetes, coronary heart disease, and history of stroke. Initially, $15.5 \%$ of men reported permanent stress (due to working conditions or personal life). During the study, 899 men developed type 2 diabetes. The scientists were able to show that subjects with permanent stress have a $45 \%$ risk in addition to developing diabetes compared to those who reported no stress. This relationship remains significant even after adjustment by age, status or physical inactivity. This work demonstrates that stressful living conditions can increase the risk of developing type 2 diabetes.

\section{Oxidative Stress Pathway and Diabetes}

Patients with diabetes have a state of oxidative stress, that is, an imbalance between reactive oxygen species and antioxidant defenses, in favor of the former.

This oxidative stress, which is accompanied by the formation of advanced glycation products (AGE), is implicated in the occurrence of diabetic complications. It may therefore be particularly advantageous to propose antioxidant and / or anti-EFA molecules in these patients as a complementary therapeutic. The antioxidants may be conventional molecules such as vitamin E, lipoic acid or $\mathrm{N}$-acetylcysteine.

Thus, treatment with vitamin E may improve the action of insulin and glycemic control, as shown by lowering blood glucose and values of glycated hemoglobin and fructosamine. In addition, this supplementation decreases plasma lipid peroxidation as well as the oxidability of low density lipoprotein (LDL), a phenomenon involved in the process of atherogenesis. This type of supplementation also makes it possible to combat the complications of the retinopathy type.

A second category of molecules is those that are capable of opposing the effects of advanced glycation products (AGEs).

They may act either:

- By preventing the cellular action of AGEs, through antagonism at the specific receptors (RAGE); This is the case with soluble RAGE;

- Or by inhibiting the formation of AGEs by the trapping of reactive carbonyl intermediates.

Nucleophilic compounds such as pyridoxamine, tenilsetam, 2,3diaminophenazone, OPB-9195 or aminoguanidine are capable of performing this action. Aminoguanidine has been shown to limit the development of major complications of diabetes in animals. A double-blind trial was conducted in the United
States and Canada in type 2 diabetics to investigate the effect of aminoguanidine on the progression of diabetic nephropathy.

A third category of molecules consists of oral antidiabetics with antioxidant properties. These are thiazolidinediones (troglitazone) and sulfonylureas (gliclazide). Thus, troglitazone and gliclazide are able to decrease the susceptibility of LDL to oxidation but also the adhesion of monocytes to endothelial cells, an early stage in the process of atherogenesis and stimulated by oxidized LDL. Finally, a future pathway is represented by oral anti-diabetics with both anti-oxidant and anti-EFA properties. Thus, a widely used oral anti-diabetic, metformin or dimethylbiguanide, helps prevent the complications of diabetes not only by lowering blood glucose, but also by inhibiting the formation of AGE and by stimulating antioxidant defenses. This last therapeutic pathway represents a future pathway in the field of diabetes, aimed at obtaining both better glycemic control and less diabetic complications.

It seems confirmed that abnormally high concentrations of glucose in extracellular and intracellular media induce oxidative stress. Several mechanisms are involved: auto-oxidation of glucose, glycation of proteins and the pathway of polyols.

A using different marker, oxidative stress was found in type 1 or type 2 diabetic patients. However, the intensity of this stress seems to depend on the type of Diabetes and control of glycemic control.

The importance of oxidative stress in the complications of diabetes remains to be better appreciated. This is a complex problem as these complications may also be due to the Advanced Protein Glycation (EFA) end products.

These two processes are highly entangled. Investigations are needed to assess their respective shares in the development and progression of diabetes complications such as retinopathy, nephropathy and neuropathy. Depending on this oxidative stress, the advantage of antioxidant supplements can be understood.

\section{Management Clues}

Managing a chronic disease can be challenging. Hence the importance of developing effective management strategies to ensure health, balance and happiness.

Type 1 diabetes is one of the most common chronic conditions in children and requires treatment that is both 


\section{Mental Health \& Human Resilience International Journal}

complex and demanding. If diabetes in children is primarily type 1 , more and more adolescents develop type 2 diabetes, which requires similar treatment, and are subject to comparable stressors.

Frequent insulin administration, glycemic control, and treatment of hyperglycemia and hypoglycemia are all part of daily life with diabetes.

Children with diabetes and their families must also coordinate these tasks with diet and physical activity. Diabetes management also includes other aspects: regular appointments in clinics and hospitals for diabetes care, monitoring of risks and treatment of medical complications, and active maintenance of the healthy immune system to prevent infections.

These challenging tasks can be disruptive and stressful and cause children with diabetes and their families to be subjected to a series of stresses that do not suffer children who do not suffer from the condition.

Living with diabetes can be painful for both parents and children because of the constant vigilance required to ensure optimum care.

Diabetes in children implies in principle determining the carbohydrate content and the dose of insulin to be taken before consuming any food, waiting for the effects and correcting any problems with values that are too high or too low.

Moreover, as children grow up, they may not be able to participate at all in social activities, or to a limited extent, because of their condition. Some children are not allowed to sleep with friends and are excluded from birthday parties and summer camps due to fears of other parents and adults.

Parents of children with diabetes sometimes feel compelled to report to their child's school at the beginning of the year, and to maintain awareness throughout the year by speaking with teachers, principals and School nurses.

Many children say they feel lonely and different from their classmates. The experiences reported are diverse: some children have a network of friends and comrades who support them, while others are tyrannized and mockery.

Like many other chronic medical conditions, diabetes requires both care from a professional team and active family participation. The person with diabetes must follow a diet, respect the rules on self-medication, performing glycemic checks regularly, practice sport and sustained regularly in clinics specialists. These people need help to achieve all these tasks.

DAWN study [6] is the most important study carried out on psychological aspects of the diabetes. It examines the points and attitudes of more than of 5,000 people with diabetes and 3,000 Health care professionals in 13 countries (Germany, Australia, Denmark, France, Germany, India, Japan, Netherlands, Norway, Poland, United Kingdom, Sweden, USA).

DAWN shows that the prevalence of depression is twice as high in these individuals, compared to the general population, or about 15 to $20 \%$, regardless of the type of diabetes.

More subtle problems, including family stress, add to these obvious stressors. In young children, parents often manage diabetes and, therefore, parenting often includes call-backs and supervision of all tasks related to the condition. Parents make every effort to ensure to their child the same fulfillment as to another, while ensuring his / her safety and health today and in the future. But even so, children sometimes feel "harassed" or more punished by their parents than their brothers and sisters. This can lead to growing disputes and tensions between parents and young people. Tensions may also arise with siblings, who sometimes feel that parents are concerned only with the child with diabetes. In quality of life studies, family members stress the limitations and anxiety associated with living with a person with diabetes taking all of these elements into account is not surprising that children with diabetes are at a greater risk of developing depression, anxiety and psychological problems than those who do not suffer from the condition.

\section{References}

1. DSM-5

2. P Grimaldi (2009) The relationship between depression and diabetes. Diabetic Medicine.

3. (2009) Annals of Family Medicine.

4. (2010) Diabetes Care.

5. JP Sauvanet (2010) Depressive state and diabetes: effect of the characteristics of depression. American Journal of Psychiatry.

6. F Snoeck (2002) DAWN study, diabetes voice. 


\section{Mental Health \& Human Resilience International Journal}

(c) (1) 